\title{
Enhancement of the File Transfer Rate in Bluetooth Sensor Systems using Base 128 secure encryption scheme and FFHS methodology
}

\author{
Abhilash Owk \\ School of Computing \\ SASTRA University
}

\author{
Anil Kuncham \\ School of Computing \\ SASTRA University
}

\author{
Harish R \\ School of Computing \\ SASTRA University
}

\begin{abstract}
Muthukumaran A
School of Computing

SASTRA University
\end{abstract}

\begin{abstract}
In this paper, we propose a new encryption algorithm for communication of Bluetooth Sensor Systems. It is well known that Bluetooth sensor systems are commonly used in low range data transfer and communication applications where security and data transfer speed are the primary concerns. Several algorithms were proposed in this regard. Analyzing the strengths and weaknesses of the current encryption algorithms, we develop a new 128 bit encryption scheme which is designed only for the Bluetooth data transfer scenario by taking advantage of the Fast Frequency Hopping Scheme and thereby achieving greater data transfer speed and security.
\end{abstract}

The full text of the article is not available in the cache. Kindly refer the IJCA digital library at www.ijcaonline.org for the complete article. In case, you face problems while downloading the full-text, please send a mail to editor at editor@ijcaonline.org 